\title{
Calculation of a three-layer plate by the finite element method taking into account the creep of the filler
}

\author{
Anton Chepurnenko, ${ }^{1, *}$, Andrei Savchenko ${ }^{1}$, and Svetlana Yazyeva ${ }^{1}$ \\ ${ }^{1}$ Don State Technical University, 344022 Sotsialisticheskaya 162, Russia
}

\begin{abstract}
In the article the derivation of the resolving equations for the calculation of a three-layer plate taking into account the creep of the middle layer by the finite element method is given. Rectangular finite elements are used. The problem reduces to a system of linear algebraic equations. An example of calculating a three-layer plate hinged on the contour and loaded with a uniformly distributed load is considered. A comparison of the results with the solution based on the finite difference method is presented.
\end{abstract}

\section{Introduction}

Three-layer structures are widely used in many industries, including aircraft construction, shipbuilding, civil and industrial engineering, etc. Such structures, as a rule, consist of two outer layers with high mechanical characteristics (steel, aluminium, fiberglass) and a lightweight filler located between them. Porous polymers (foams) are widely used as a filler. For polymers in addition to elastic properties, a pronounced rheology is characteristic. The calculation of threelayered structures taking the creep into account is considered in [1-3]. Resolving equations for a triangular finite element of a three-layer plate and shell are given in [1-2]. In the present paper rectangular finite elements with higher accuracy will be considered.

\section{Derivation of resolving equations}

The rectangular finite element of the three-layer plate is shown in Fig. 1-2. Each node of this element has 5 degrees of freedom: displacements in the plane of the upper layer $u_{i}^{-}$and $v_{i}^{-}$, displacements in the plane of the lower layer $u_{i}^{+}$and $v_{i}^{+}$, as well as deflection $w_{i}$.

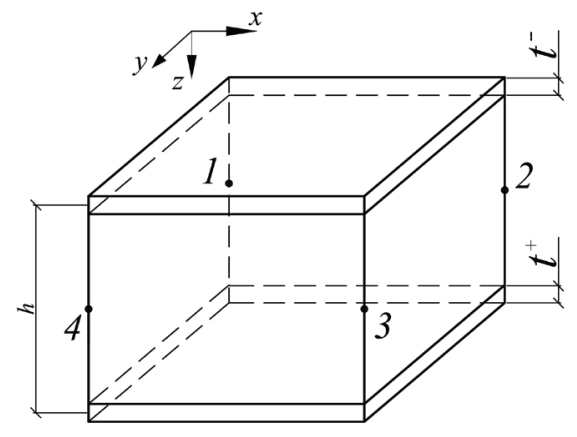

Fig. 1. Rectangular finite element of a three-layer plate: general view.

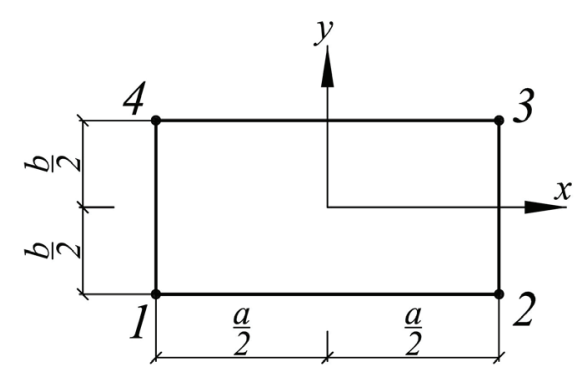

Fig. 2. Rectangular finite element in the plane.

For the displacement field within the element, we take the following approximation:

$$
\begin{aligned}
& u^{+(-)}=N_{1} u_{1}^{+(-)}+N_{2} u_{2}^{+(-)}+N_{3} u_{3}^{+(-)}+N_{4} u_{4}^{+(-)} \\
& v^{+(-)}=N_{1} v_{1}^{+(-)}+N_{2} v_{2}^{+(-)}+N_{3} v_{3}^{+(-)}+N_{4} v_{4}^{+(-)} \\
& w=N_{1} w_{1}+N_{2} w_{2}+N_{3} w_{3}+N_{4} w_{4},
\end{aligned}
$$

where $N_{1}, N_{2}, N_{3}, N_{4}$ - form functions.

$$
\begin{aligned}
& N_{1}=\frac{1}{a b}\left(\frac{a}{2}-x\right)\left(\frac{b}{2}-y\right) ; \quad N_{2}=\frac{1}{a b}\left(\frac{a}{2}+x\right)\left(\frac{b}{2}-y\right) \\
& N_{3}=\frac{1}{a b}\left(\frac{a}{2}+x\right)\left(\frac{b}{2}+y\right) ; \quad N_{4}=\frac{1}{a b}\left(\frac{a}{2}-x\right)\left(\frac{b}{2}+y\right)
\end{aligned}
$$

where $a, b$-dimensions of the finite element.

The coordinates $x$ and $y$ in formulas (2) are measured from the centre of the finite element.

The deformation vector of a finite element is written as:

$$
\{\varepsilon\}=\left\{\begin{array}{llllllll}
\varepsilon_{x}^{+} & \varepsilon_{y}^{+} & \gamma_{x y}^{+} & \varepsilon_{x}^{-} & \varepsilon_{y}^{-} & \gamma_{x y}^{-} & \gamma_{z x}^{m} & \gamma_{y x}^{m}
\end{array}\right\}^{T},
$$

where $\varepsilon_{x}^{+}, \varepsilon_{y}^{+}, \gamma_{x y}^{+}-$deformations of the lower layer, $\varepsilon_{x}^{-}, \varepsilon_{y}^{-}, \gamma_{x y}^{-}-$deformations of the upper layer, $\gamma_{z x}^{m}$, $\gamma_{y x}^{m}$-deformations of the filler.

\footnotetext{
* Corresponding author: anton_chepurnenk@mail.ru
} 
In the technical theory of three-layer plates, the relationship between displacements and deformations has the form:

$$
\begin{gathered}
\varepsilon_{x}^{+(-)}=\frac{\partial u^{+(-)}}{\partial x} ; \\
\varepsilon_{y}^{+(-)}=\frac{\partial v^{+(-)}}{\partial y} ; \\
\gamma_{x y}^{+(-)}=\frac{\partial u^{+(-)}}{\partial y}+\frac{\partial v^{+(-)}}{\partial x} ; \\
\gamma_{z x}^{m}=\frac{u^{+}-u^{-}}{h}+\frac{\partial w}{\partial x} \\
\gamma_{z y}^{m}=\frac{v^{+}-v^{-}}{h}+\frac{\partial w}{\partial y} .
\end{gathered}
$$

Substituting (1) in (3), we obtain the following relationship between the node displacements and deformations in the matrix form:

$$
\{\varepsilon\}=[B]\{U\},
$$

where $\{U\}$ - vector of nodal displacements.

$$
\{U\}=\left\{\begin{array}{l}
\left\{\rho_{1}\right\} \\
\left\{\rho_{2}\right\} \\
\left\{\rho_{3}\right\} \\
\left\{\rho_{4}\right\}
\end{array}\right\}, \quad\left\{\rho_{i}\right\}=\left\{\begin{array}{lllll}
u_{i}^{+} & v_{i}^{+} & u_{i}^{-} & v_{i}^{-} & w_{i}
\end{array}\right\}^{T} .
$$

Matrix $[B]$ has a size of $8 \times 20$. Non-zero elements of the matrix $[B]$ are defined as follows:

$$
\begin{aligned}
& B_{1,1}=B_{3,2}=B_{4,3}=B_{6,4}=B_{7,5}=\frac{\partial N_{1}}{\partial x} ; B_{1,6}=B_{3,7}= \\
& =B_{4,8}=B_{6,9}=B_{7,10}=\frac{\partial N_{2}}{\partial x} ; B_{1,11}=B_{3,12}=B_{4,13}= \\
& =B_{6,14}=B_{7,15}=\frac{\partial N_{3}}{\partial x} ; B_{1,16}=B_{3,17}=B_{4,18}=B_{6,19}= \\
& =B_{7,20}=\frac{\partial N_{4}}{\partial x} ; B_{2,2}=B_{3,1}=B_{5,4}=B_{6,3}=B_{8,5}=\frac{\partial N_{1}}{\partial y} ; \\
& B_{2,7}=B_{3,6}=B_{5,9}=B_{6,8}=B_{8,10}=\frac{\partial N_{2}}{\partial y} ; B_{2,12}= \\
& B_{3,11}=B_{5,14}=B_{6,13}=B_{8,15}=\frac{\partial N_{3}}{\partial y} ; B_{2,17}=B_{3,16}= \\
& =B_{5,19}=B_{6,18}=B_{8,20}=\frac{\partial N_{4}}{\partial y} ; B_{7,1}=B_{8,2}=-B_{7,3}= \\
& =-B_{8,4}=\frac{N_{1}}{h} ; B_{7,6}=B_{8,7}=-B_{7,8}=-B_{8,9}=\frac{N_{2}}{h} ; \\
& B_{7,11}=B_{8,12}=-B_{7,13}=-B_{8,14}=\frac{N_{3}}{h} ; B_{7,16}=B_{8,17}= \\
& =-B_{7,18}=-B_{8,19}=\frac{N_{4}}{h} .
\end{aligned}
$$

The partial derivatives of the form functions are written as:

$$
\begin{aligned}
& \frac{\partial N_{1}}{\partial x}=-\frac{1}{a b}\left(\frac{b}{2}-y\right) ; \quad \frac{\partial N_{2}}{\partial x}=\frac{1}{a b}\left(\frac{b}{2}-y\right) ; \\
& \frac{\partial N_{3}}{\partial x}=\frac{1}{a b}\left(\frac{b}{2}+y\right) ; \quad \frac{\partial N_{4}}{\partial x}=-\frac{1}{a b}\left(\frac{b}{2}+y\right) ; \\
& \frac{\partial N_{1}}{\partial y}=-\frac{1}{a b}\left(\frac{a}{2}-x\right) ; \quad \frac{\partial N_{2}}{\partial y}=-\frac{1}{a b}\left(\frac{a}{2}+x\right) ; \\
& \frac{\partial N_{3}}{\partial y}=\frac{1}{a b}\left(\frac{a}{2}+x\right) ; \quad \frac{\partial N_{4}}{\partial y}=\frac{1}{a b}\left(\frac{a}{2}-x\right) .
\end{aligned}
$$

The resolving equations will be obtained using the variational principle of Lagrange. The potential energy of deformation of a three-layer plate considering creep is defined as follows:

$$
\begin{aligned}
& W=\frac{1}{2} \int_{A} t^{+}\left(\sigma_{x}^{+} \varepsilon_{x}^{+}+\sigma_{y}^{+} \varepsilon_{y}^{+}+\tau_{x y}^{+} \gamma_{x y}^{+}\right)+ \\
& +t^{-}\left(\sigma_{x}^{-} \varepsilon_{x}^{-}+\sigma_{y}^{-} \varepsilon_{y}^{-}+\tau_{x y}^{-} \gamma_{x y}^{-}\right)+ \\
& +h\left[\tau_{z x}^{m}\left(\gamma_{z x}^{m}-\gamma_{z x}^{m *}\right)+\tau_{z y}^{m}\left(\gamma_{z y}^{m}-\gamma_{z y}^{m *}\right)\right] d A,
\end{aligned}
$$

where $\gamma_{z x}^{m *}, \gamma_{z y}^{m *}$ - creep deformations of the filler, $A-$ area of the finite element.

Expression (4) can be rewritten as:

$W=\frac{1}{2} \int_{A}\{N\}^{T}\left(\{\varepsilon\}-\left\{\varepsilon^{*}\right\}\right) d A=\frac{1}{2} \int_{A}\{N\}^{T}\left([B]\{U\}-\left\{\varepsilon^{*}\right\}\right) d A$, where $\quad\left\{\varepsilon^{*}\right\}=\left\{\begin{array}{llllllll}0 & 0 & 0 & 0 & 0 & 0 & \gamma_{z x}^{m *} & \gamma_{z y}^{m *}\end{array}\right\}^{T}-$ creep strain vector,

$\{N\}^{T}=\left\{\begin{array}{llllllll}N_{x}^{+} & N_{y}^{+} & N_{x y}^{+} & N_{x}^{-} & N_{y}^{-} & N_{x y}^{-} & Q_{z x} & Q_{z y}\end{array}\right\}$

- vector of internal forces, $N_{x}^{+(-)}=\sigma_{x}^{+(-)} \cdot t^{+(-)}$,

$$
N_{y}^{+(-)}=\sigma_{y}^{+(-)} \cdot t^{+(-)}, \quad N_{x y}^{+(-)}=\tau_{x y}^{+(-)} \cdot t^{+(-)},
$$

$Q_{z x}=\tau_{z x}^{m} \cdot h, Q_{z y}=\tau_{z y}^{m} \cdot h$.

The relationship between deformations and internal forces has the form:

$$
\{N\}=[D]\left(\{\varepsilon\}-\left\{\varepsilon^{*}\right\}\right)=[D]\left([B]\{U\}-\left\{\varepsilon^{*}\right\}\right),
$$

where $[D]-$ block matrix of elastic constants.

$$
\begin{gathered}
{[D]=\left[\begin{array}{ccc}
{\left[D^{+}\right]} & & \\
& {\left[D^{-}\right]} & \\
& & {\left[D^{m}\right]}
\end{array}\right],} \\
{\left[D^{+(-)}\right]=\frac{E t^{+(-)}}{1-v^{2}}\left[\begin{array}{ccc}
1 & v & 0 \\
v & 1 & 0 \\
0 & 0 & (1-v) / 2
\end{array}\right], \quad\left[D^{m}\right]=G_{m} h\left[\begin{array}{ll}
1 & 0 \\
0 & 1
\end{array}\right],} \\
G_{m}-\text { shear modulus of the filler. }
\end{gathered}
$$


Substituting (6) in the expression for the potential energy, we get:

$$
\begin{aligned}
& W=\frac{1}{2}\left(\{U\}^{T} \int_{A}[B]^{T}[D][B] d A\{U\}-\{U\}^{T} \int_{A}[B]^{T}[D] d A\left\{\varepsilon^{*}\right\}-\right. \\
& \left.-\left\{\varepsilon^{*}\right\}^{T} \int_{A}[D][B] d A\{U\}+\int_{A}\left\{\varepsilon^{*}\right\}^{T}[D]\left\{\varepsilon^{*}\right\} d A\right)= \\
& =\frac{1}{2}\{U\}^{T} \int_{A}[B]^{T}[D][B] d A\{U\}-\{U\}^{T} \int_{A}[B]^{T}[D] d A\left\{\varepsilon^{*}\right\}+ \\
& +\frac{1}{2} \int_{A}\left\{\varepsilon^{*}\right\}^{T}[D]\left\{\varepsilon^{*}\right\} d A .
\end{aligned}
$$

The total energy represents the difference between the potential energy of deformation and the potential of external forces:

$$
T=W-A,
$$

where $A=\{U\}^{T}\{F\},\{F\}$ - vector of external nodal forces.

Differentiating the total energy with respect to the vector of nodal displacements, we obtain:

$$
\frac{\partial T}{\partial\{U\}}=[K]\{U\}-\{F\}-\left\{F^{*}\right\}=0,
$$

where $\quad[K]=\int_{A}[B]^{T}[D][B] d A-\quad$ stiffness matrix, $\left\{F^{*}\right\}=\int_{A}[B]^{T}[D] d A\left\{\varepsilon^{*}\right\}$ - the contribution of creep strains to the right-hand side of the system of linear algebraic FEM equations.

Exact expressions for the matrix $[K]$ and vector $\left\{F^{*}\right\}$ coefficients were obtained using functions for the symbol variables of the Matlab package. The vector $\left\{F^{*}\right\}$ has the form:

$$
\left\{F^{*}\right\}=\left\{\begin{array}{c}
\left\{F_{1}\right\} \\
-\frac{G_{m} h}{2}\left(a \gamma_{z y}^{m *}+b \gamma_{z x}^{m *}\right) \\
\left\{F_{1}\right\} \\
\frac{G_{m} h}{2}\left(-a \gamma_{z y}^{m *}+b \gamma_{z x}^{m *}\right) \\
\left\{F_{1}\right\} \\
\frac{G_{m} h}{2}\left(a \gamma_{z y}^{m *}+b \gamma_{z x}^{m *}\right) \\
\left\{F_{1}\right\} \\
\frac{G_{m} h}{2}\left(a \gamma_{z y}^{m *}-b \gamma_{z x}^{m *}\right)
\end{array}\right\},
$$

where $\left\{F_{1}\right\}=\frac{a b G_{m}}{4}\left\{\begin{array}{c}\gamma_{z x}^{m *} \\ \gamma_{z y}^{m *} \\ -\gamma_{z x}^{m *} \\ -\gamma_{z y}^{m *}\end{array}\right\}$.
The stiffness matrix is not presented here due to its bulkiness.

\section{Results and discussion}

A three-layer rectangular hinged plate (Fig. 3) was calculated with the following initial data: plate thickness $h=8 \mathrm{~cm}$, modulus of elasticity of sheaths $E=2 \cdot 10^{5}$ $\mathrm{MPa}$, Poisson's coefficient of sheaths $v=0.3$, sheath thickness $t^{+}=t^{-}=1.5 \mathrm{~mm}$, shear modulus of the filler $G_{m}=2.5 \mathrm{MPa}$, dimensions of plate $a=b=3 \mathrm{~m}$. The uniformly distributed over the area load $q=2 \mathrm{kPa}$ acts on the plate. As the law of creep, the equation of the linear theory of heredity was used:

$$
G_{m} \gamma_{i}=\tau_{i}+\int_{-\infty}^{t} \tau_{i} K(t-\tau) d \tau, \quad i=(x z, y z)
$$

The core of creep was assumed to be exponential:

$$
K(t-\tau)=C_{m} e^{-\alpha_{m}(t-\tau)} ; \quad C_{m}=\alpha_{m}=0,077 \frac{1}{\text { hour }} \text {. }
$$

With an exponential kernel, the creep law (6) is easily represented in a differential form:

$$
G_{m} \frac{\partial \gamma_{i}}{\partial t}+G_{m} \alpha_{m} \gamma_{i}=\frac{\partial \tau_{i}}{\partial t}+\left(\alpha_{m}+C_{m}\right) \tau_{i}
$$

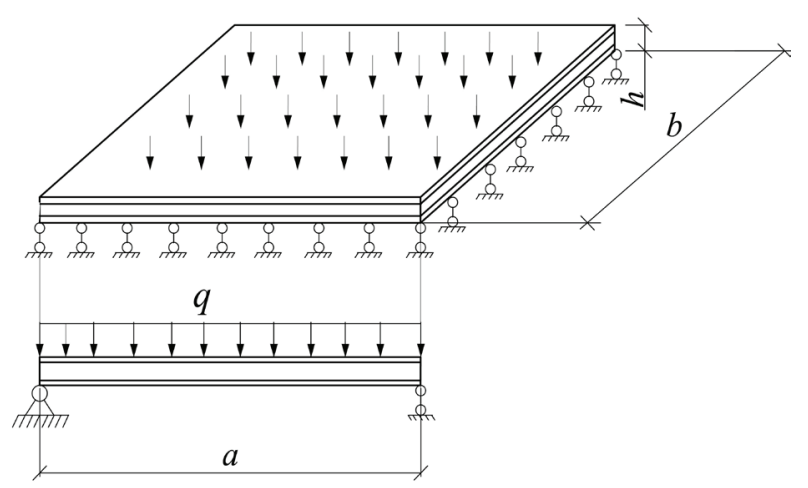

Fig. 3. Settlement scheme of the plate

Expressions (6) and (7) contain the values of the total shear deformation of the filler, which represent the sum of elastic deformations and creep strains:

$$
\gamma_{i}=\frac{\tau_{i}}{G_{m}}+\gamma_{i}^{*} .
$$

Using (8), we can express from (7) the growth rates of creep strains:

$$
\frac{\partial \gamma_{i}^{*}}{\partial t}=\frac{C_{m}}{G_{m}} \tau_{i}-\alpha_{m} \gamma_{i}^{*}
$$

The calculation was carried out by the step method, the creep strains at the time $t+\Delta t$ were determined as follows:

$$
\gamma_{i}^{*}(t+\Delta t)=\gamma_{i}^{*}(t)+\frac{\partial \gamma_{i}^{*}(t)}{\partial t} \Delta t
$$


This method is also used in [4-9]. The following boundary conditions were assumed:

$$
\begin{aligned}
& x=0, x=a: \quad w=0, \quad v^{+}=v^{-}=0 ; \\
& y=0, y=b: w=0, \quad u^{+}=u^{-}=0 \text {. }
\end{aligned}
$$

Fig. 4 shows the curve of growth of the deflection in the center of the plate. The solid line corresponds to the solution obtained by the authors using the finite element method, the dashed line is the solution by the finite difference method according to the method described in [10]. When $t=0$ the results are the same and at $t \rightarrow \infty$ the difference is $1.83 \%$.

Stresses in the skins and filler do not change during creep. The stress $\sigma_{x}^{+}$and $\tau_{x y}^{+}$distribution in the lower skin is shown in Fig. 5 - 6. The stresses in the upper skin under the boundary conditions (10) in absolute value coincide with the stresses in the lower skin. The distribution of tangential stresses in the filler is shown in Fig. 7.

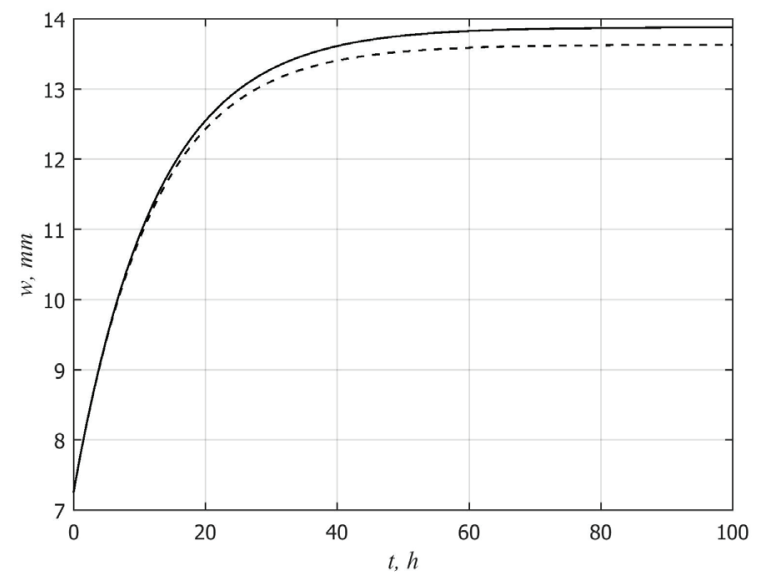

Fig. 4. Graph of growth of deflection in the center of the plate: solid line - finite element method, dashed line - finite differences method.

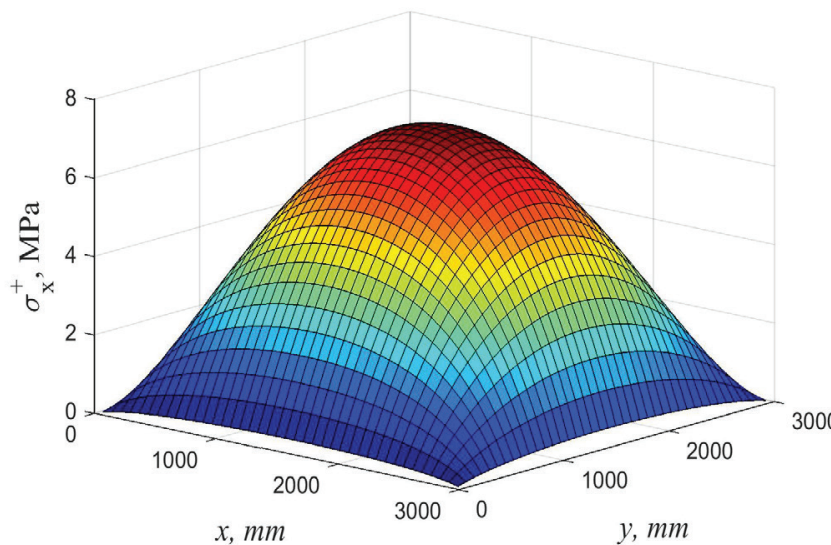

Fig. 5. Distribution of normal stresses in the lower skin.

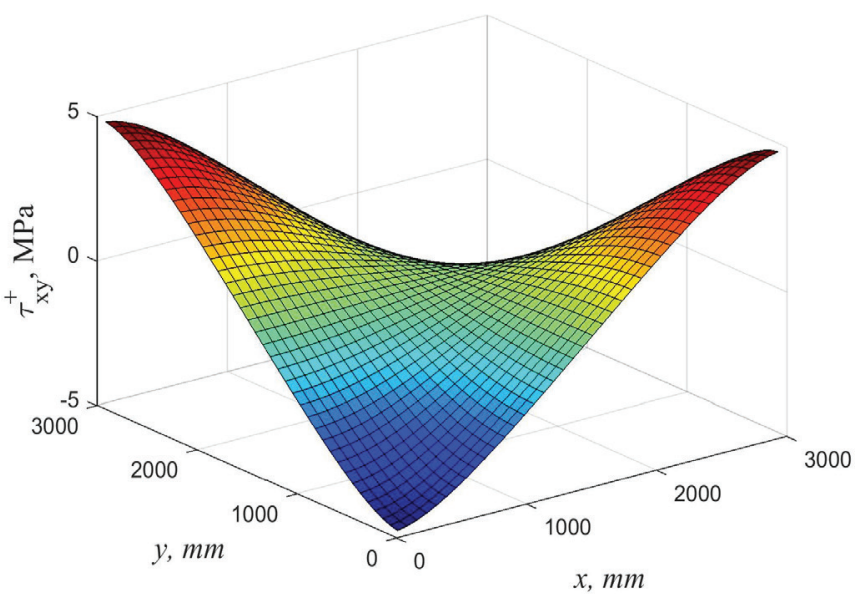

Fig. 6. The distribution of tangential stresses in the bottom skin.

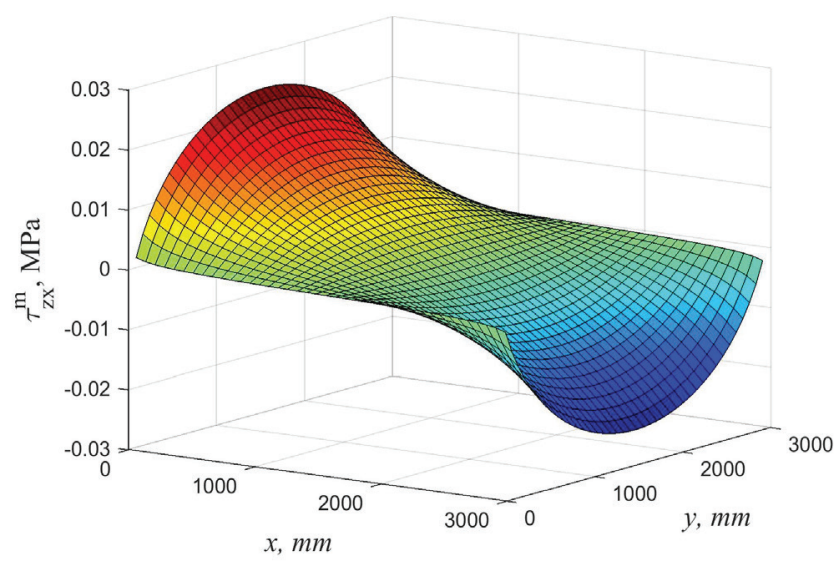

Fig. 7. Distribution of tangential stresses in the filler.

\section{Conclusions}

The equations obtained are applicable for arbitrary creep laws of the filler, including nonlinear ones. The correctness of the equations and the reliability of the results are confirmed by a comparison with the solution on the basis of the finite difference method. It is established that under linear creep law the stresses in the shells and filler do not change during the creep process.

\section{References}

1. A. Chepurnenko, L. Mailyan, B. Jazyev, Procedia Engineering, 165, pp. 990 - 994 (2016)

2. V. Andreev, B. Yaziev, A. Chepurnenko, S. Litvinov, Vestnik MGSU, 7, pp. 17-24 (2015)

3. B. Yaziev, A. Chepurnenko, S. Litvinov, S. Yaziev, Bulletin of the Dagestan State Technical University, 2, pp.47-55 (2014)

4. S. Litvinov, L. Trush, S. Yazyev, Procedia Engineering, 150, pp. 1686-1693 (2016)

5. S. Litvinov, E. Klimenko, I. Kulinich, S. Yazyeva, International Polymer Science and Technology, 2, pp. 23-25 (2015) 
6. S. Litvinov, L. Trush, A. Dudnik, Engineering Bulletin of the Don, 2, 2016. http://www.ivdon.ru/en/magazine/archive/n2y2016/ $\underline{3560}$

7. V. Andreev, A. Chepurnenko, B. Yazyev, Advanced Materials Research, 1004-1005, pp. 257-260 (2014)

8. V. Andreev, E. Barmenkova, Applied Mechanics and Materials, 204-208, pp. 3596-3599 (2012)

9. V. Andreev, A. Avershyev, WIT Transactions on the Built Environment. Fluid Structure Interaction VII , pp. 123-132 (2013)

10. V. Andreev, B. Yazyev, A. Chepurnenko, Advanced Materials Research, 900, pp. 707-710 (2014) 